\title{
From Coordination to Collapse in Rigged Economies
}

\author{
A game-theoretical model of a rigged economy predicts the emergence of \\ cartels followed by a risk of instability as the economy becomes more \\ complex.
}

By Sitabhra Sinha

6 'The economy is rigged!" This claim, which was voiced by both Bernie Sanders and Donald Trump during their 2016 presidential campaigns, might be the only belief shared by people from opposite ends of the political spectrum. But what does "rigging" mean for the economy and its dynamics? Luís Seoane at the National Center for Biotechnology in Spain has now addressed this question by modeling the economy as a system of "games" that agents can rig-for a price [1]. The study reveals that the rigged economy undergoes a sequence of transitions as its complexity and size increase, with "cartels" forming and then dissolving. Although these transitions appear to imply that economic development will ultimately make the

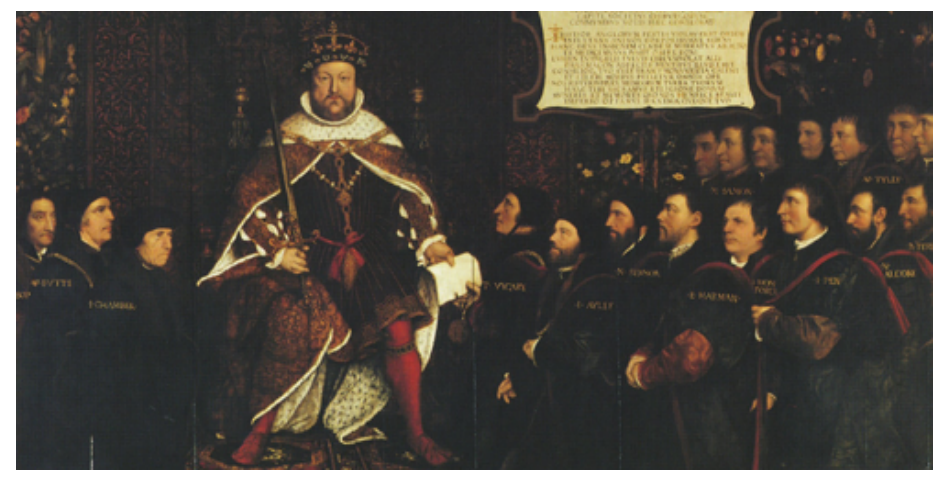

Figure 1: King Henry VIII and the Barber Surgeons by Hans Holbein the Younger. Medieval guilds played negative as well as positive roles by rigging the economy: They acted as cartels to privilege their members while at the same time working in concert to promote property rights and to prevent the arbitrary use of power by monarchs.

Credit: Hans Holbein (1543) economy fairer, Seoane shows that if an economy's size does not keep pace with its rising complexity, large fluctuations in wealth distribution can occur, causing inequality to rise steeply and making the economy liable to collapse.

The use of games to study economic phenomena dates back to the work of John von Neumann in the middle of the last century [2]. The theory quickly became the lingua franca for economists and subsequently emerged as an area of inquiry for the physics community. For example, statistical physicists have shown that versions of the "minority game"-in which several agents choose between two possibilities, with the option chosen by fewest agents becoming the winning choice-can be used to explore the rich emergent properties of simple adaptive systems [3].

Minority games can be used to model situations in which agents compete for scant resources-including financial markets. However, the economy is also marked by phenomena in which the advantage lies with those in the majority, for example, when positive feedback reinforces a particular choice, such as joining a boycott [4]. Seoane shows that rigged economies exhibit features of both minority and majority games $[5,6]$.

In Seoane's model, multiple agents engage in a number of games simultaneously. Each game involves choosing one of two possible actions. An agent can also choose to pay to rig a game to favor its choice. The winning choice in each round is the action chosen by the majority of the agents who have paid to intervene. Increasing the number of games played in each round increases the degrees of freedom in the economy and is thus a measure of its complexity. After each round of a game, 
the winners share a fixed amount of money equally. The value of the winnings multiplied by the number of games played defines the total "wealth" that can be redistributed among the agents in each round. This wealth is thus a measure of the size of the economy and, along with complexity, is a key parameter of the model.

To observe how the optimal strategy for an agent changes as the economy develops, Seoane includes in the model an evolutionary process: Agents can replicate themselves after each round, such that each offspring has a high probability of adopting the same strategy as its parent. Since replication costs a fixed amount, more successful agents reproduce in greater numbers.

Seoane observes that, for a fixed level of complexity, a small economy yields agents with diverse strategies and a general preference to be in the minority when they win (to claim more of the prize pot in each round). As the economy increases in size, more wealth becomes available for agents to create progeny, as well as to pay the intervention costs required to rig games. Then, agents switch from playing minority games to majority games, meaning growing economies transition to coordination between agents (cartel formation), with an accompanying drop in strategic diversity. However, if the complexity (the number of games per round) increases faster than the economy grows, the relative returns per game are diminished, causing agents to seek minority positions across multiple games. This switch leads to the dissolution of cartels and a rise in the diversity of strategies employed by the agents.

Seoane also studies how an economy fares under other size-complexity relations. For example, he finds that when the amount disbursed in each game is constant, such that the size of the economy increases linearly with the number of games, there is a critical "complexity threshold" at which the distribution of agent-population size transitions from unimodal to bimodal. This transition gives rise to extremely large fluctuations in agent populations that threaten the stability of the economy. The distribution of agent wealth also exhibits a crossover at this point, becoming broad-tailed in the large-fluctuation regime, indicating rising inequality among agents. A reader familiar with the world's economy today will likely see eerie parallels with this regime of the model.
It could be argued that some of the outcomes observed by Seoane come from the specific choices he makes in constructing the model. For example, the transition to coordinated action as the economy grows might result from the assumption that intervention costs are constant. In reality, intervention costs are related to the size of the economy and vary between players. Another important limitation of the model is that all agents are equally able to rig games-an assumption that misses the asymmetric influence of the wealthy in real economies [7].

Even with these limitations, Seoane's model is significant in that it provides a framework for others to explore the ramifications of real-world rigged economies, such as those in which information is unevenly distributed among agents. Modifying the model might, for example, reveal how informationally disadvantaged agents can use the emergence of coordination to obtain advantage, as seen in other agent-based models [8]. Such counter-rigging of the system by less powerful players is shown by the history of the medieval merchant guilds. Those institutions used the threat of coordinated embargo to resist arbitrary expropriation by powerful local rulers [9], proving that a rigged economy does not necessarily imply that David doesn't stand a chance against Goliath.

Sitabhra Sinha: The Institute of Mathematical Sciences, Chennai, India

\section{REFERENCES}

1. L. F. Seoane, "Games in rigged economies," Phys. Rev. X 11, 031058 (2021).

2. J. von Neumann and O. Morgenstern, Theory of games and economic behavior (Princeton University Press, Princeton, 1944).

3. D. Challet et al., Minority games: Interacting agents in financial markets (Oxford University Press, New York, 2005).

4. T. C. Schelling, Micromotives and macrobehavior (W. W. Norton, New York, 1978).

5. J. Vitting Andersen and D. Sornette, "The \$-game," Eur. Phys. J. B 31, 141 (2003).

6. Y. Baek et al., "Market behavior and performance of different strategy evaluation schemes," Phys. Rev. E 82, 026109 (2010).

7. J. E. Stiglitz, "The American economy is rigged," Sci. Am. 319, 56 (2018).

8. V. Sasidevan et al., "When big data fails: Adaptive agents using coarse-grained information have competitive advantage," Phys. 
Rev. E 98, 020301 (2018).

9. A. Greif et al., "Coordination, commitment, and enforcement:
The case of the merchant guild," J. Polit. Econ. 102, 745 (1994). 